\title{
Acetonitrile extract of Salvia miltiorrhiza Radix exhibits growth-inhibitory effects on prostate cancer cells through the induction of cell cycle arrest and apoptosis
}

\author{
$\mathrm{JUNE}_{\mathrm{LEE}}{ }^{1}, \mathrm{BU}$ YOUNG $\mathrm{CHOI}^{2}$ and YOUNG-SAM KEUM ${ }^{1}$ \\ ${ }^{1}$ College of Pharmacy, Dongguk University, Goyang, Gyeonggi 410-820; ${ }^{2}$ Department of \\ Pharmaceutical Science and Engineering, Seowon University, Cheongju, Chungcheongbuk 361-742, Republic of Korea
}

Received January 17, 2016; Accepted December 2, 2016

DOI: $10.3892 / \mathrm{ol} .2017 .5820$

\begin{abstract}
In the present study, the effects of the acetonitrile or water extracts from 400 selected traditional medicinal plants on the growth of PC-3 cells were investigated, and it was demonstrated that an acetonitrile extract of Salvia miltiorrhiza Radix exhibited the most marked cytotoxic effects on PC-3 cells. It was observed that the acetonitrile extract of S. miltiorrhiza Radix induced marked cell cycle arrest and apoptosis in PC-3 cells through the generation of intracellular reactive oxygen species. It was also demonstrated that oral administration of the acetonitrile extract of $S$. miltiorrhiza Radix decreased the incidence and growth of PC-3 tumor xenografts in nude mice. The results of the present study suggest that the acetonitrile extract of $S$. miltiorrhiza Radix exhibits marked inhibitory effects on the growth of prostate cancer cells in vitro and in vivo.
\end{abstract}

\section{Introduction}

Prostate cancer is a primary public health problem in Western countries, according to the 2014 cancer statistics, and represents the most commonly diagnosed type of cancer and second leading cause of cancer-associated male death in the USA (1). It is well-known that primary prostate tumors respond poorly to high dosages of chemotherapeutic agents or radiotherapy (2). Testosterone is the major circulating androgen, therefore patients with advanced prostate cancer often undergo androgen-deprivation therapy (ADT) that directly decreases the endogenous testosterone level through a radical prostatectomy and/or inhibition of the androgen receptor activity using pharmacological inhibitors (3). However, therapeutic benefits

Correspondence to: Professor Young-Sam Keum, College of Pharmacy, Dongguk University, 32 Dongguk-ro, Goyang, Gyeonggi 410-820, Republic of Korea

E-mail: keum03@dongguk.edu

Key words: Salvia miltiorrhiza Radix, prostate cancer, cell cycle arrest, apoptosis, reactive oxygen species from ADT are frequently short-lived because castration-resistant and incurable tumors may emerge in patients with prostate cancer (4).

Numerous chemical agents or nutritional supplements have been reported to exhibit significant prostate chemopreventative activities in experimental animal models (5). Therefore, a number of population-based studies were performed to examine the potential prostate chemopreventative effects of selected natural or synthetic compounds (6). In contrast with initial expectations, however, none of the compounds produced meaningful chemopreventative effects that were able to be translated into the clinical setting (7), suggesting that the identification of novel medications that were able to inhibit the growth of prostate cancer cells remains of clinical importance. The aim of the present study was to identify novel medicinal plant extracts that may exert inhibitory effects on the growth of prostate cancer, and an acetonitrile extract of Salvia miltiorrhiza Radix was demonstrated to exhibit marked suppressive effects on the growth of prostate cancer cells.

\section{Materials and methods}

Cell culture, chemicals and reagents. All traditional medicinal plant extracts used in the present study were purchased from the Korean Plant Extract Bank (Ochang, South Korea). RPMI-1640 medium, heat-inactivated fetal bovine serum (FBS), penicillin/streptomycin and PBS were purchased from Welgene (Gyeongsan, South Korea). PC-3, LNCaP and DU-145 prostate cancer cells were purchased from the Korean Cell Line Bank (Seoul, South Korea) and cultured in RPMI-1640 medium, containing 10\% heat-inactivated FBS and $1 \mathrm{X}$ penicillin and streptomycin at $37^{\circ} \mathrm{C}$ in a humidified $5 \% \mathrm{CO}_{2}$ incubator. Dimethyl sulfoxide (DMSO), N-acetylcysteine (NAC), bromodeoxyuridine (BrdU) and horseradish peroxidase (HRP)-conjugated secondary antibodies (catalog nos. sc-2031 and sc-2054) were purchased from Santa Cruz Biotechnology, Inc. (Dallas, TX, USA). Paraformaldehyde, the bicinchoninic acid (BCA) protein assay kit and polyvinylidene fluoride (PVDF) membranes were purchased from EMD Millipore (Billerica, MA, USA). The DeadEnd ${ }^{\mathrm{TM}}$ fluorimetric terminal deoxynucleotidyltransferase-mediated dUTP nick end labeling (TUNEL) system kit was purchased 
from Promega Corporation (Madison, WI, USA). Propidium iodide (PI), trypan blue solution, 2',7'-dichlorofluorescin diacetate (DCF-DA) and antibodies against BrdU (catalog no. sc-32323), 8'-hydroxyguanosine (8-OH-G) and $\beta$-actin were purchased from Sigma-Aldrich; Merck Millipore (Darmstadt, Germany). All other primary antibodies related to cell cycle arrest and apoptosis analysis [Cell Cycle Regulation Sampler kit (catalog no. 9932) or Apoptosis Antibody Sampler kit (catalog no. 9915)] were purchased from Cell Signaling Technology, Inc. (Danvers, MA, USA). Fluorescein isothiocyanate (FITC)-conjugated secondary antibody was purchased from Jackson ImmunoResearch Laboratories, Inc. (West Grove, PA, USA). Matrigel ${ }^{\mathrm{TM}}$ basement membrane matrix was purchased from BD Biosciences (Franklin Lakes, NJ, USA).

MTT assay. PC- 3 cells ( $3 \times 10^{4}$ cells/ $/ 100 \mu 1 /$ well) were plated in 96-well culture plates. Following incubation at $37^{\circ} \mathrm{C}$ in a humidified $5 \% \mathrm{CO}_{2}$ incubator with separate acetonitrile and water extracts (data not shown) of 400 traditional medicinal plants at a concentration of $20 \mu \mathrm{g} / \mathrm{ml}$ for $24 \mathrm{~h}$, cells were mixed with $50 \mu \mathrm{l}$ MTT stock solution $(2 \mathrm{mg} / \mathrm{ml})$ for $4 \mathrm{~h}$ at $37^{\circ} \mathrm{C}$. PC-3 cells were washed with $1 \mathrm{X}$ PBS and lysed with $50 \mu \mathrm{l}$ DMSO, followed by spectrophotometric measurement at $540 \mathrm{~nm}$. The percentage of viable cells was determined in comparison with the control group. Cells were also pretreated with $10 \mu \mathrm{M}$ NAC for $24 \mathrm{~h}$.

Trypan blue exclusion assay. LNCaP, DU-145 and PC-3 prostate cancer cells $\left(3 \times 10^{5}\right.$ cells $/ 1 \mathrm{ml} /$ well in quadruplicate) were plated in 6-well culture plates and exposed to various concentrations $(5,20$ and $100 \mu \mathrm{g} / \mathrm{ml})$ of the acetonitrile extract of S. miltiorrhiza Radix for 24 or 48 h. Following washing with 1X PBS, cells were harvested by centrifugation at $800 \mathrm{x} g$ for $5 \mathrm{~min}$ at room temperature, and stained with $0.4 \%$ trypan blue solution for $3 \mathrm{~min}$ at room temperature. The number of viable cells was determined using a light microscope and a hemocytometer.

BrdU incorporation assay. PC- 3 cells $\left(7.5 \times 10^{4}\right.$ cells on a cover glass) were exposed to $\mathrm{BrdU}(10 \mu \mathrm{M})$ alone or in combination with the acetonitrile extract of S. miltiorrhiza Radix $(20 \mu \mathrm{g} / \mathrm{ml})$ for 6 or $12 \mathrm{~h}$. Following fixation with 4\% paraformaldehyde for $15 \mathrm{~min}$ at room temperature, cells were washed once with $1 \mathrm{X}$ immunocytochemistry washing buffer $(0.1 \%$ Triton X-100 and $1 \%$ horse serum in PBS). Cells were then hybridized with primary antibody against BrdU (1:400 dilution) overnight at $4^{\circ} \mathrm{C}$, followed by hybridization at $4^{\circ} \mathrm{C}$ with an FITC-conjugated secondary antibody (1:1,000 dilution) for $1 \mathrm{~h}$. The final fluorescent image was captured using a C2 confocal microscope (Nikon Korea, Seoul, South Korea).

TUNEL assay. PC-3 cells ( $7.5 \times 10^{4}$ cells on a coverslip) were exposed to the acetonitrile extract of $S$. miltiorrhiza Radix $(20 \mu \mathrm{g} / \mathrm{ml})$ for $6 \mathrm{~h}$, and a TUNEL assay was conducted using the DeadEnd ${ }^{\mathrm{TM}}$ fluorimetric TUNEL system kit, according to the manufacturer's protocol.

Measurement of cell cycle arrest and intracellular reactive oxygen species (ROS) levels by fluorescence-activated cell sorting (FACS). Following exposure to the acetonitrile extract of S. miltiorrhiza Radix (20 $\mu \mathrm{g} / \mathrm{ml})$ for 24,48 or $72 \mathrm{~h}$, PC-3 cells were washed with $1 \mathrm{X}$ PBS and harvested. Equal numbers of cells $\left(1 \times 10^{6} / \mathrm{group}\right)$ were dispensed into glass tubes and fixed for $1 \mathrm{~h}$ at $37^{\circ} \mathrm{C}$ with a solution containing $1 \mathrm{X}$ PBS and $70 \%$ ethanol. Following washing with $1 \mathrm{X}$ PBS, cells were mixed with PI $(20 \mu \mathrm{g} / \mathrm{ml})$ or DCF-DA $(1 \mu \mathrm{M})$, and the cell cycle changes and intracellular ROS levels were measured using a FACSCalibur flow cytometer and CellQuest Pro software (BD Biosciences).

Western blot analysis. Following exposure to the acetonitrile extract of S. miltiorrhiza Radix $(20 \mu \mathrm{g} / \mathrm{ml})$ alone or in combination with $10 \mu \mathrm{M}$ NAC for $>48 \mathrm{~h}, \mathrm{PC}-3$ cells were collected by scraper and centrifugation at $10,000 \times \mathrm{x} g$ at room temperature. Cells were then lysed in $200 \mu l$ radioimmunoprecipitation assay buffer (50 mM Tris- $\mathrm{HCl}$ (pH 8.0), $150 \mathrm{mM} \mathrm{NaCl}, 1 \% \mathrm{NP}-40$, $0.5 \%$ sodium deoxycholate and protease inhibitor cocktail (catalog no. P3100; GenDepot, Seoul, Korea)] on ice for $1 \mathrm{~h}$. Cell lysates were collected and protein concentrations were determined using the BCA protein assay kit (catalog no. 23227; Pierce; Thermo Fisher Scientific, Inc. Waltham, MA, USA), according to the manufacturer's protocol. Equal amounts $(30 \mu \mathrm{g})$ of cell lysates were resolved using 8-12\% SDS-PAGE and transferred onto PVDF membranes. The membranes were incubated in blocking buffer [5\% skimmed milk in PBST (1X PBS and 0.1\% Tween-20)] for $1 \mathrm{~h}$ at $4^{\circ} \mathrm{C}$ and hybridized with the aforementioned primary antibodies (1:1,000 dilution) in 1X PBS containing 3\% bovine serum albumin (BSA; GenDepot, Katy, TX, USA) or 3\% skimmed milk overnight at $4^{\circ} \mathrm{C}$. Following washing three times with $1 \mathrm{X}$ PBST for $30 \mathrm{~min}$, the membrane was hybridized with anti-mouse and anti-rabbit HRP-conjugated secondary antibodies (1:5,000 dilution) for $1 \mathrm{~h}$ at room temperature and washed three times with 1X PBST solution for $30 \mathrm{~min}$. The membrane was visualized using an enhanced chemiluminescence detection system (Pierce; Thermo Fisher Scientific, Inc.) according to the manufacturer's instructions. $\beta$-actin was used as a loading control.

Measurement of 8-OH-Glevels. In order to measure changes in 8 -OH-G levels, PC-3 cells (75,000 cells/well in $500 \mu 1$ media) grown on coverslips were treated with the acetonitrile extract of S. miltiorrhiza Radix ( $20 \mu \mathrm{g} / \mathrm{ml})$ for $6 \mathrm{~h}$ alone or in combination with $1 \mathrm{mM}$ NAC, and incubated with blocking serum (1\% BSA) for $30 \mathrm{~min}$ at room temperature. Following washing three times with 1X PBS, cells were hybridized with primary antibody against $8-\mathrm{OH}-\mathrm{G}$ (1:400 dilution) overnight at $4^{\circ} \mathrm{C}$. Following washing three times with $1 \mathrm{X}$ PBS, the coverslips were probed with FITC-conjugated rabbit secondary antibody (1:1,000 dilution) at room temperature. The fluorescent images were captured using a $\mathrm{C} 2$ confocal microscope.

PC-3 xenograft study. A total of 32 6-week-old, male, immunodeficient mice (negative control group, 16 mice with a mean weight of $19.9 \mathrm{~g}$; and for oral administration, 16 mice with a mean weight of $19.7 \mathrm{~g}$ ) were purchased from Daehan Biolink Co., Ltd. (Eumseong, South Korea). Animals were housed in sterile filter-capped microisolator cages under a $12 \mathrm{~h} \mathrm{light} / 12 \mathrm{~h}$ dark cycle, and provided with water and an AIN-76 diet ad libitum. After 1 week of acclimation, individual mice were subcutaneously injected into the right flank with PC-3 cells 
$\left(2 \times 10^{6}\right.$ cells $/ 0.2 \mathrm{ml}$ suspension medium), which were previously suspended with 50\% Matrigel $^{\mathrm{TM}}$ in ice-cold RPMI-1640 medium. The acetonitrile extract of $S$. miltiorrhiza Radix (100 $\mathrm{mg} / \mathrm{kg}$ in PBS) was administered by oral gavage for 6 weeks, and the incidence and size of tumors were measured using a caliper at the end of each week. Tumor volume $(V)$ was calculated as $V=\left(L \times W^{2}\right) \times 0.52$, where $L$ is the length and $W$ is the width of a xenograft during the course of the experiment. At sacrifice by asphyxiation with $\mathrm{CO}_{2}$, tumors were excised and weighed. All animal experiments were performed under an Institutional Animal Care and Use Committee-approved protocol (IACUC-2013-003) from Dongguk University (Seoul, South Korea).

Statistical analysis. Statistical analysis was performed by Student's $t$-test using Microsoft Excel 2013 (Microsoft Corporation, Redmond, WA, USA). Results are presented as the mean \pm standard deviation. $\mathrm{P}<0.05$ was considered to indicate a statistically significant difference.

\section{Results}

Acetonitrile extract of $S$. miltiorrhiza Radix exhibits significant suppressive effects on the growth of prostate cancer cells. The aim of the present study was to identify novel traditional medicinal plant extract(s) that exhibit marked inhibitory effects on the growth of prostate cancer cells. PC-3 cells were exposed to 400 medicinal plant extracts, prepared using water or acetonitrile, at a concentration of $20 \mu \mathrm{g} / \mathrm{ml}$ for $24 \mathrm{~h}$, and an MTT assay was performed to measure the cell viability. The results demonstrated that, whereas none of the water extracts of traditional medicinal plants affected the growth of PC-3 cells (data not shown), the acetonitrile extract of S. miltiorrhiza Radix exerted a marked inhibitory effect on the growth of PC-3 cells (Fig. 1). In order to acquire the exact cell viability, various concentrations of the acetonitrile extract of $S$. miltiorrhiza Radix were exposed to three prostate cancer cell lines (LNCaP, DU-145 and PC-3 cells) and a trypan blue exclusion assay was performed. The results demonstrated that the acetonitrile extract of $S$. miltiorrhiza Radix significantly inhibited the growth of all three cell lines in a dose- and time-dependent manner compared with the untreated control cells $(\mathrm{P}<0.05 ;$ Fig. 2A). In addition, exposure to the acetonitrile extract of $S$. miltiorrhiza Radix inhibited BrdU incorporation (Fig. 2B) and elicited marked cell death (Fig. 2C) in PC-3 cells compared with the control. These results indicate that the acetonitrile extract of $S$. miltiorrhiza Radix exhibited inhibitory effects on the growth of prostate cancer cells.

Acetonitrile extract of S. miltiorrhiza Radix induces cell cycle arrest and apoptosis in PC-3 cells. To determine whether the acetonitrile extract of $S$. miltiorrhiza Radix may induce cell cycle arrest, PC-3 cells were exposed to the acetonitrile extract of S. miltiorrhiza Radix for 24, 48 and $72 \mathrm{~h}$, and cell cycle changes were assessed using FACS analysis. It was observed that the acetonitrile extract of $S$. miltiorrhiza Radix significantly induced cell cycle arrest at G1/S phase compared with the untreated control $(\mathrm{P}<0.01$; Fig. $3 \mathrm{~A})$. Western blot analysis demonstrated that the acetonitrile extract of $S$. miltiorrhiza Radix increased the expression of $\mathrm{p} 21$ protein and decreased cyclin-dependent kinase 2 (CDK2), CDK4 and cyclin D1 protein levels (Fig. 3B). These results suggest that the induction of cell cycle arrest at G1/S phase by the acetonitrile extract of $S$. miltiorrhiza Radix may be attributable to changes in the levels of G1/S cell cycle regulator proteins.

It is well-known that the induction of cell cycle arrest at G1/S phase, also referred to as a cell cycle checkpoint, temporarily allows cells to repair the cellular damage caused by extracellular or intracellular stress (8). Alternatively, the cell cycle checkpoint may also activate signaling pathways, leading to apoptosis if cellular damage is extensive and unable to be properly repaired (9). Therefore, it was investigated whether exposure to the acetonitrile extract of $S$. miltiorrhiza Radix may induce apoptosis in PC-3 cells. It was observed that the acetonitrile extract of $S$. miltiorrhiza Radix induced apoptosis in PC-3 cells, as determined using the TUNEL assay (Fig. 3C). Western blot analysis indicated that the acetonitrile extract of S. miltiorrhiza Radix decreased the expression level of apoptosis regulator Bcl-2 and activated apoptosis-inducer proteins, including caspase-9, caspase-3 and poly (ADP-ribose) polymerase (PARP) (Fig. 3D). It should be noted that the acetonitrile extract of $S$. miltiorrhiza Radix exhibited no significant effect on the activity of caspase- 8 , a critical protein that initiates the intrinsic apoptotic signaling pathway (Fig. 3D). These results suggest that the induction of apoptosis using the acetonitrile extract of S. miltiorrhiza Radix may occur exclusively through the activation of the extrinsic apoptotic signaling pathway in PC-3 cells.

Induction of cell cycle arrest and apoptosis by the acetonitrile extract of $S$. miltiorrhiza Radix is dependent on the generation of intracellular ROS. It is known that the generation of intracellular ROS regulates various cellular processes, including cell cycle arrest and apoptosis (10). Based on the observations that the acetonitrile extract of S. miltiorrhiza Radix is a potent inducer of cell cycle arrest and apoptosis (Fig. 3), it was hypothesized that generation of intracellular ROS may be involved in these events. Consistently, it was observed that treatment with the acetonitrile extract of S. miltiorrhiza Radix induced the generation of intracellular ROS in PC-3 cells (Fig. 4A). In addition, it was observed that a combinatorial treatment of NAC, an antioxidant peptide that is used as a precursor for the synthesis of intracellular reduced glutathione thereby contributing to the detoxification of intracellular ROS (11), with the acetonitrile extract of S. miltiorrhiza Radix completely abrogated the decrease in cellular CDK4 and cyclin D1 levels that was observed in the absence of NAC (Fig. 4B). These results suggest that intracellular ROS may be responsible for cell cycle arrest at G1/S. Consistently, the MTT assay demonstrated that NAC significantly decreased the cell death induced by the acetonitrile extract of $S$. miltiorrhiza Radix compared with in the absence of NAC $(\mathrm{P}<0.05$; Fig. 4C). In addition, NAC prevented the activation of caspase- 3 and PARP proteins induced by the acetonitrile extract of $S$. miltiorrhiza Radix (Fig. 4D). It was also observed that NAC reduced the formation of intracellular 8-OH-G, an oxidative stress marker induced by the acetonitrile extract of $S$. miltiorrhiza Radix in PC-3 cells (Fig. 4E). These results suggest that the induction of cell cycle arrest and apoptosis by the acetonitrile extract 

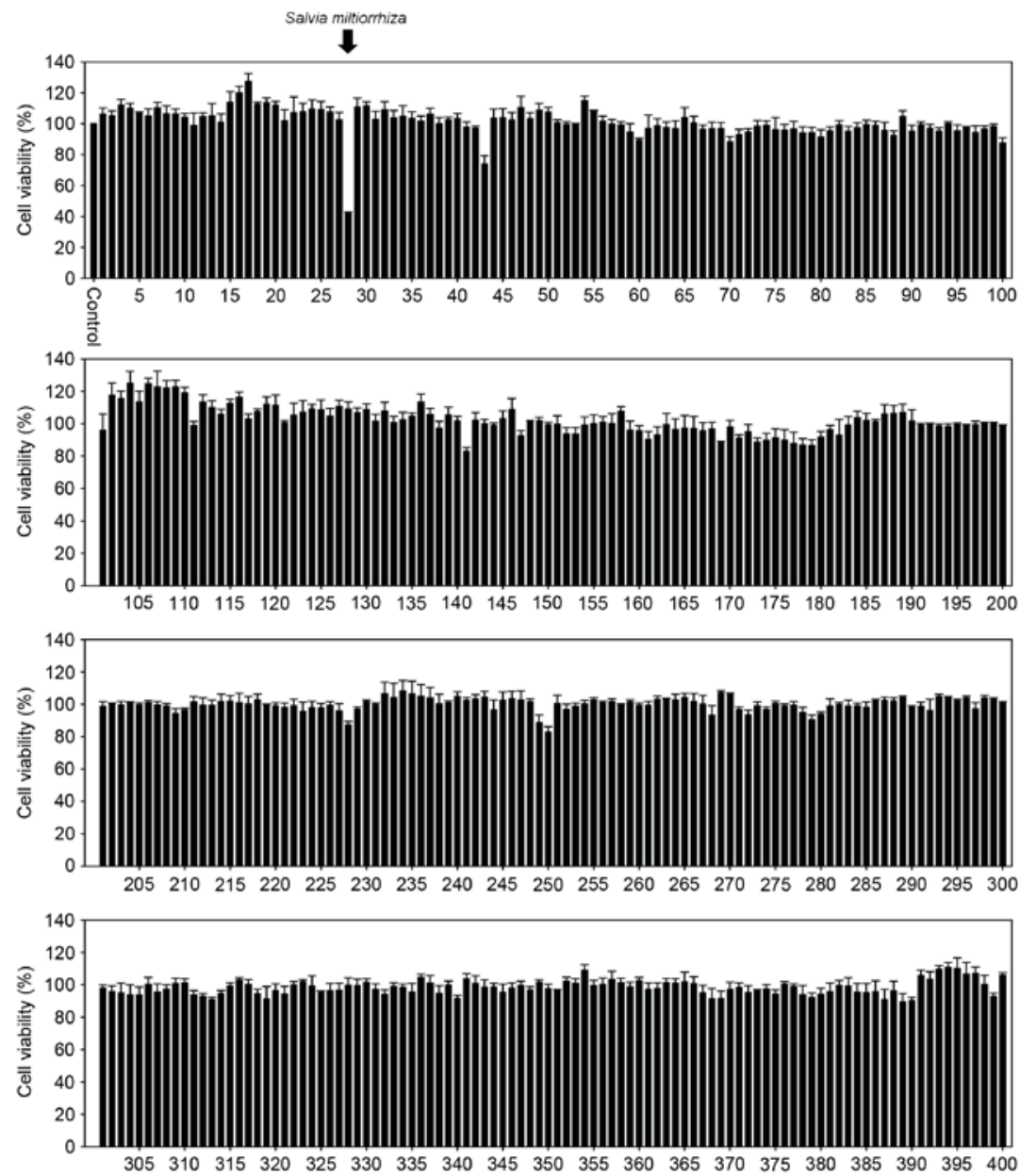

Figure 1. Measurement of PC-3 cell viability following exposure to various acetonitrile extracts of traditional medicinal plants. PC-3 cells were plated in 96-well culture plates and exposed to 400 distinct acetonitrile extracts of traditional medicinal plants for $24 \mathrm{~h}$. Cell viability was measured using an MTT assay. The acetonitrile extract from Salvia miltiorrhiza Radix was determined to have a marked effect on PC-3 cell viability.
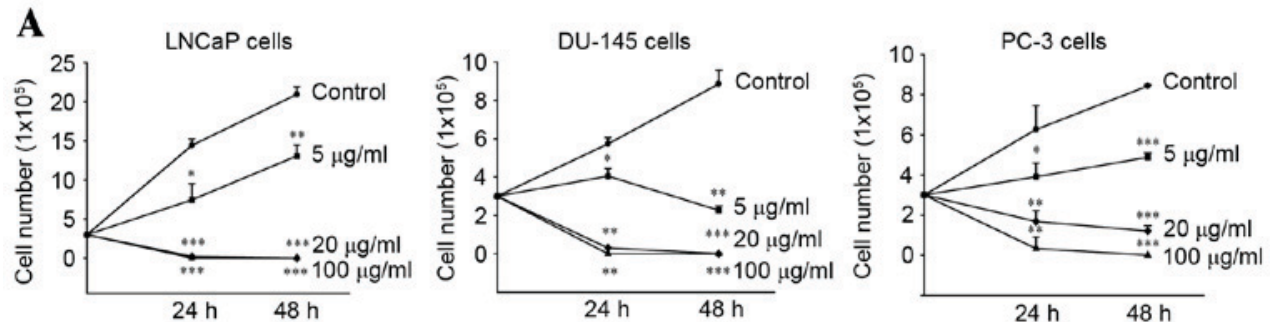

B

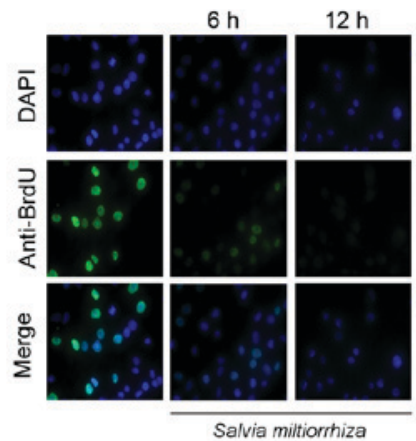

C

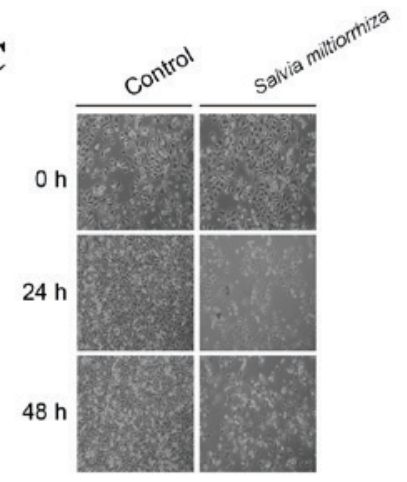

Figure 2. Acetonitrile extract of Salvia miltiorrhiza Radix inhibits the proliferation of prostate cancer cells. (A) PC-3, LNCaP and DU-145 cells were exposed to the acetonitrile extract of $S$. miltiorrhiza Radix and cell viability was measured using a trypan blue exclusion assay. ${ }^{*} \mathrm{P}<0.05,{ }^{* *} \mathrm{P}<0.01,{ }^{* * * *} \mathrm{P}<0.001 \mathrm{vs}$. control. (B) PC-3 cells were exposed to the acetonitrile extract of S. miltiorrhiza Radix and a BrdU incorporation assay was conducted. (C) PC-3 cells were exposed to the acetonitrile extract of S. miltiorrhiza Radix and the gross morphology of PC-3 cells was observed using a phase-contrast microscope. BrdU, bromodeoxyuridine. 

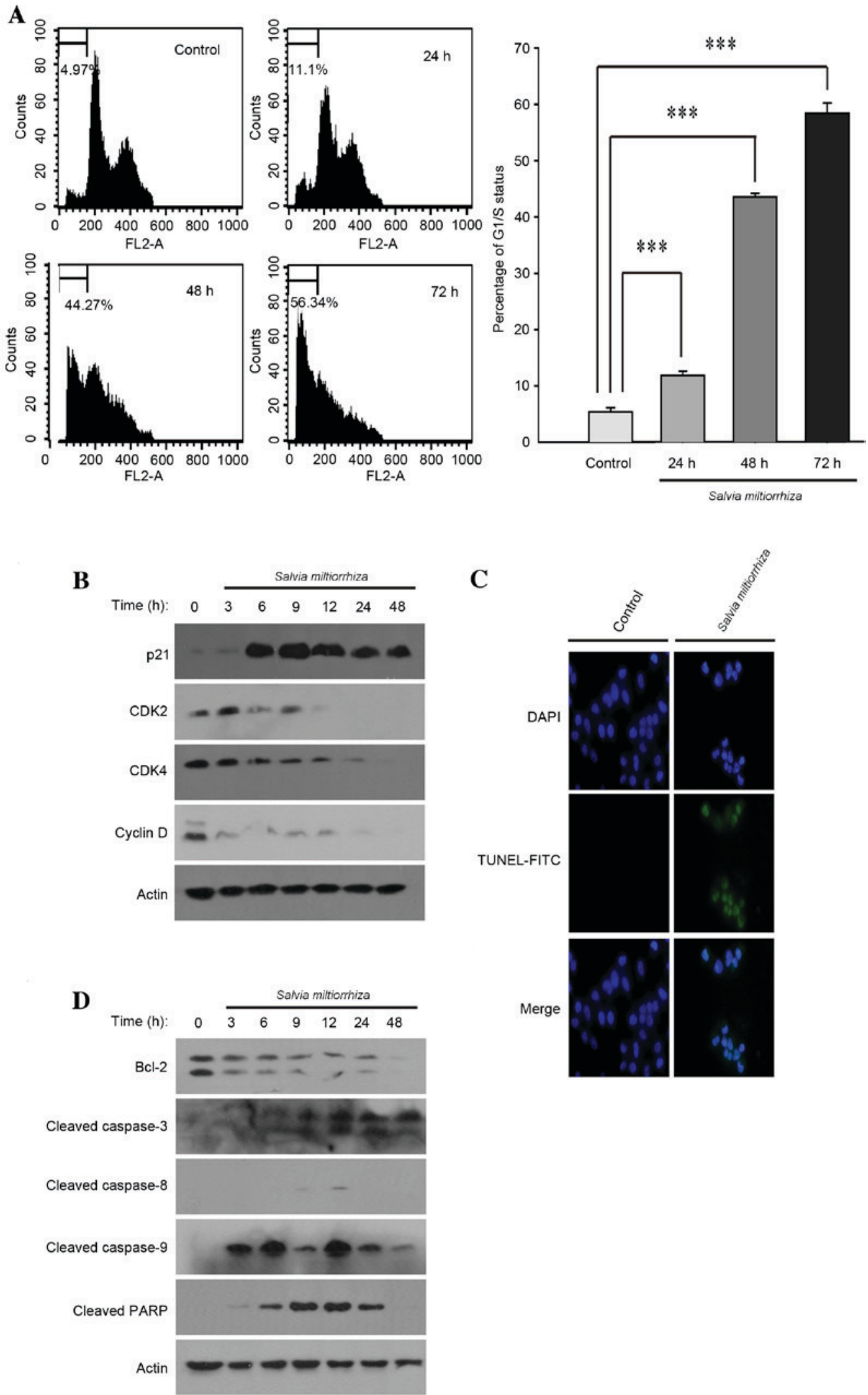

C

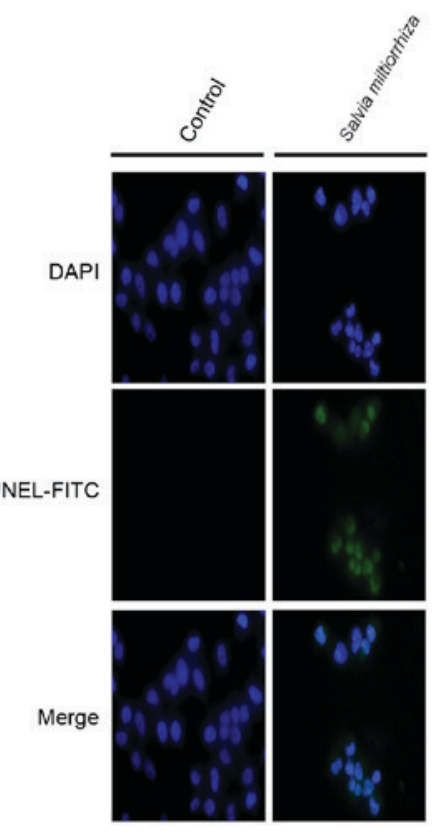

Figure 3. Acetonitrile extract of Salvia miltiorrhiza Radix induces cell cycle arrest and apoptosis in PC-3 cells. (A) PC-3 cells were exposed to the acetonitrile extract of S. miltiorrhiza Radix and the resulting cell cycle changes were monitored using fluorescence-activated cell sorting analysis (left panels). The experiments were performed in triplicate and the percentage of G1/S cell cycle arrest was analyzed and depicted (right panel). ${ }^{* * * *} \mathrm{P}<0.001$ vs. control. (B) PC-3 cells were exposed to the acetonitrile extract of S. miltiorrhiza Radix and western blot analysis was performed using polyclonal antibodies against G1/S cell cycle regulator proteins (p21, CDK2, CDK4 and cyclin D). $\beta$-Actin was used as a loading control. (C) PC-3 cells, grown on a coverslip, were exposed to the acetonitrile extract of S. miltiorrhiza Radix and a TUNEL assay was performed. Representative confocal microscopy images are presented. (D) PC-3 cells were exposed to the acetonitrile extract of S. miltiorrhiza Radix and western blot analysis was performed using polyclonal antibodies against apoptosis-related proteins (Bcl-2, cleaved caspase-8, cleaved caspase-9, cleaved caspase-3 and cleaved PARP). $\beta$-actin was used as a loading control. CDK, cyclin-dependent kinase; TUNEL, terminal deoxynucleotidyltransferase-mediated dUTP nick end labeling; FITC, fluorescein isothiocyanate; Bcl-2, apoptosis regulator; PARP, poly (ADP-ribose) polymerase. 
A

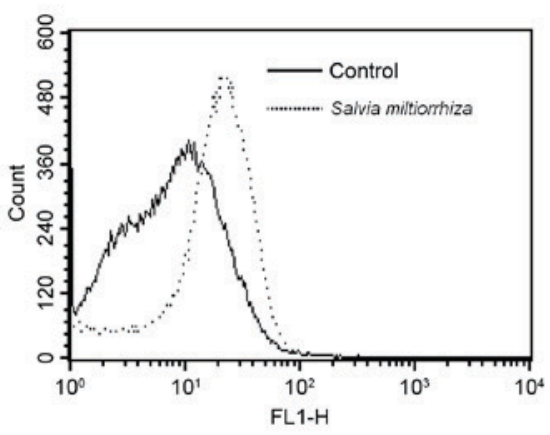

B

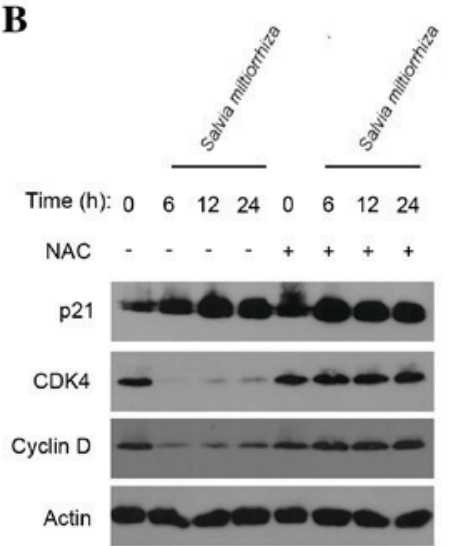

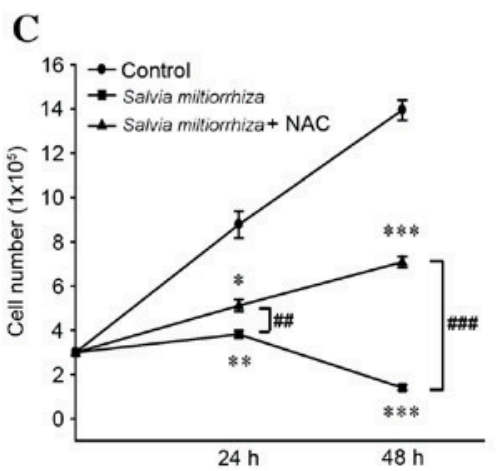

D

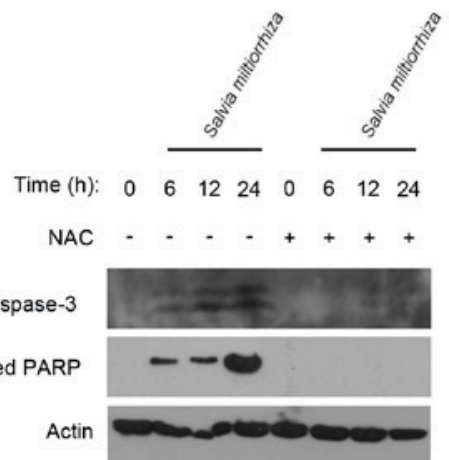

$\mathbf{E}$

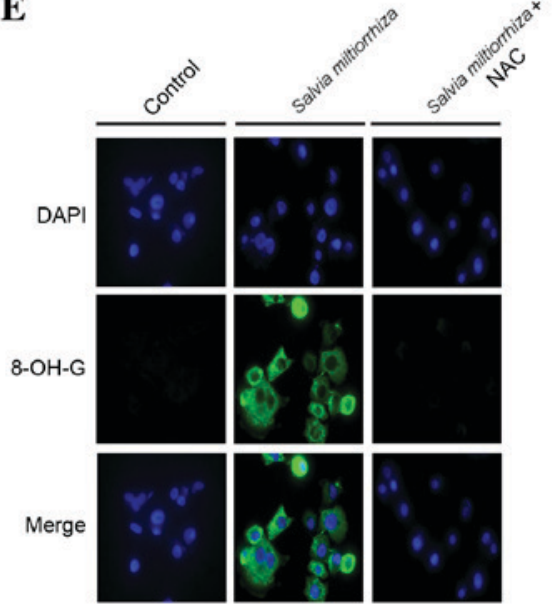

Figure 4. Induction of cell cycle arrest and apoptosis by the acetonitrile extract of Salvia miltiorrhiza Radix is dependent on the generation of intracellular ROS (A) PC-3 cells were exposed to the acetonitrile extract of S. miltiorrhiza Radix for $24 \mathrm{~h}$ and stained with 2',7'-dichlorofluorescin diacetate. The generation of intracellular ROS was monitored by fluorescence-activated cell sorting analysis. (B) PC-3 cells were exposed to the acetonitrile extract of S. miltiorrhiza Radix alone or in combination with NAC. Western blot analysis was performed using polyclonal antibodies against G1/S cell cycle regulator proteins (p21, CDK4 and cyclin D). $\beta$-Actin was used as a loading control. (C) PC-3 cells were exposed to the acetonitrile extract of S. miltiorrhiza Radix alone or in combination with NAC and an MTT assay was performed. ${ }^{*} \mathrm{P}<0.05,{ }^{* *} \mathrm{P}<0.01,{ }^{* * *} \mathrm{P}<0.001$ vs. control; ${ }^{\# \#} \mathrm{P}<0.01,{ }^{\# \# \#} \mathrm{P}<0.001$ vs. treatment with NAC. (D) PC-3 cells were exposed to the acetonitrile extract of S. miltiorrhiza Radix alone or in combination with NAC, and western blot analysis was performed using polyclonal antibodies against apoptosis-related proteins (cleaved caspase-3 and cleaved PARP). $\beta$-Actin was used as a loading control. (E) PC-3 cells, grown on a coverslip were exposed to the acetonitrile extract of S. miltiorrhiza Radix alone or in combination with NAC, and hybridized using primary antibody against 8-OH-G. Representative confocal microscopy images of the immunocytochemistry are presented. ROS, reactive oxygen species; NAC, $N$-acetylcysteine; CDK4, cyclin-dependent kinase; PARP, poly (ADP-ribose) polymerase; 8-OH-G, 8'-hydroxyguanosine.

of S. miltiorrhiza Radix is mediated by the generation of intracellular ROS.

Oral administration of acetonitrile extract of $S$. miltiorrhiza Radix suppresses the incidence and growth of PC-3 tumor xenografts in vivo. As the acetonitrile extract of S. miltiorrhiza
Radix efficiently induced cell cycle arrest and apoptosis of PC-3 cells in vitro, it was investigated whether the acetonitrile extract of $S$. miltiorrhiza Radix may suppress the growth of PC-3 cells in vivo. PC-3 cells were injected into the flank of nude mice, which were orally administered with the acetonitrile extract of S. miltiorrhiza Radix for 6 weeks (Fig. 5A). 
$\mathbf{A}$

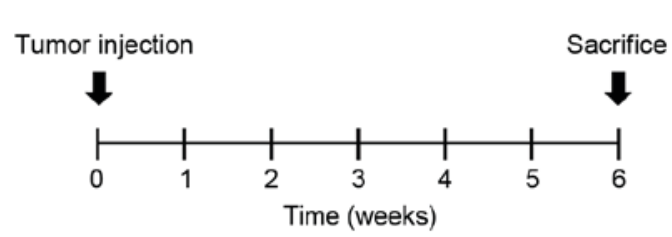

B

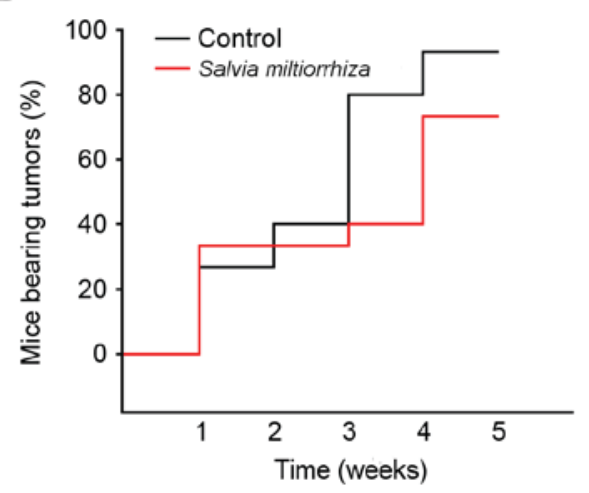

C

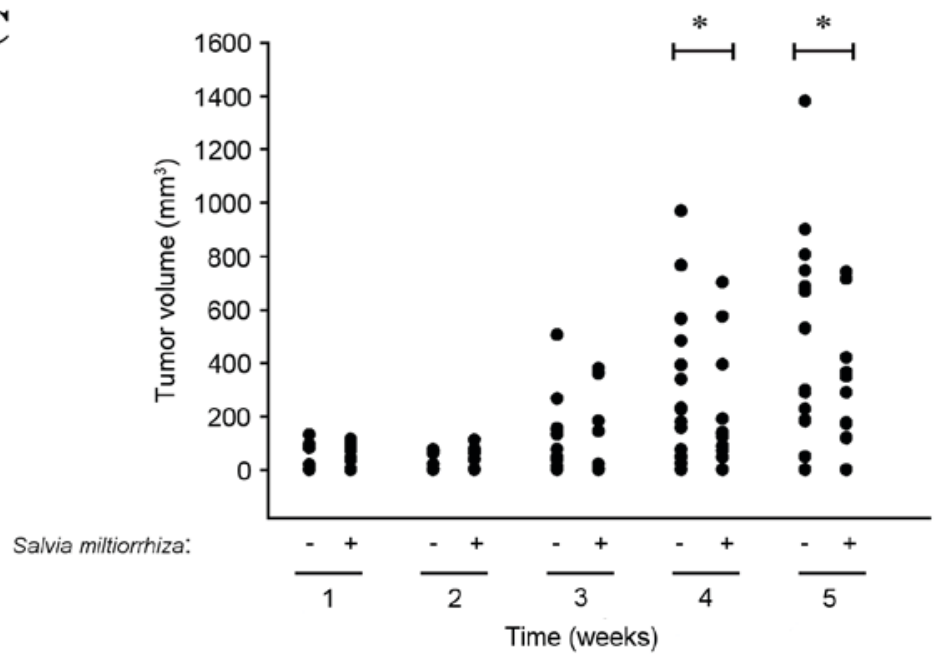

D
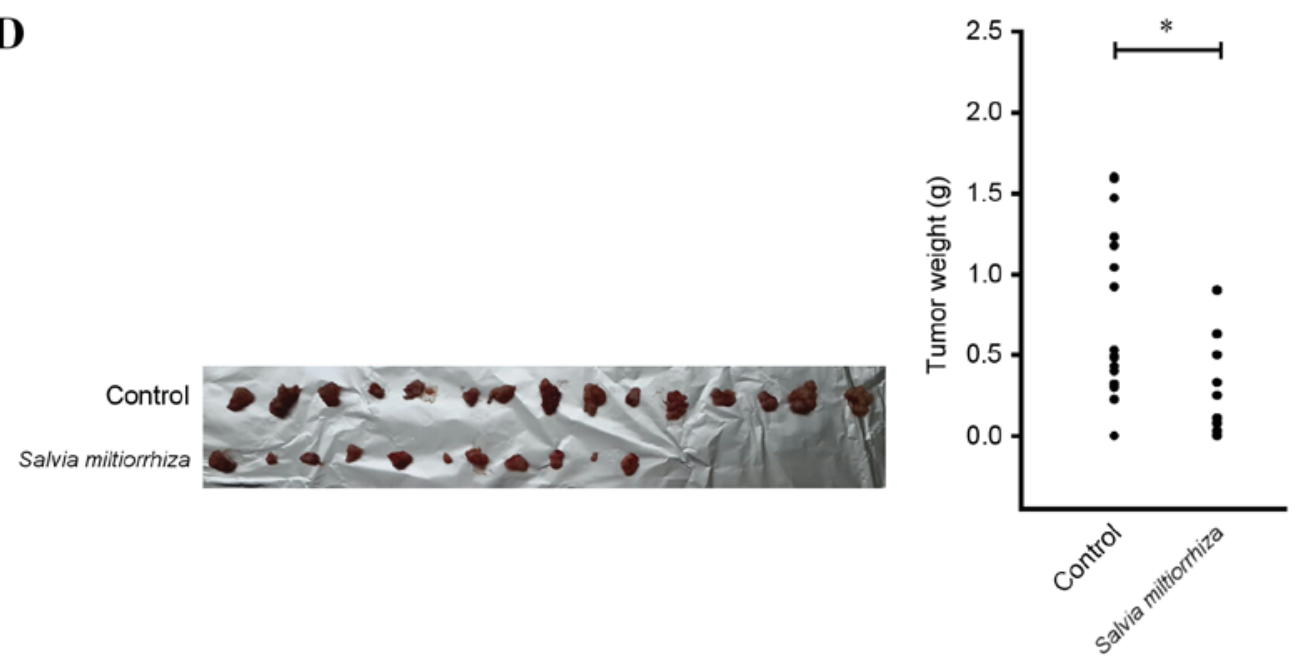

Figure 5. Acetonitrile extract of Salvia miltiorrhiza Radix suppresses the incidence and growth of PC-3 xenografts in nude mice. (A) Scheme of PC-3 xenograft experiment. Mice were randomly divided into two groups: Control group $(\mathrm{n}=16)$ and S. miltiorrhiza Radix group (n=15). After 1 week of acclimation, mice were orally fed with vehicle (1X PBS) or the acetonitrile extract of S. miltiorrhiza Radix in 1X PBS. (B) Percentage of mice bearing palpable tumors over time. (C) Measurement of PC-3 xenograft tumor size over time. (D) Tumor incidence (left panel) and weight (right panel) of PC-3 xenografts extracted from mice were determined at sacrifice. ${ }^{*} \mathrm{P}<0.05$.

It was observed that the overall body weight of mice in the group of the acetonitrile extract of S. miltiorrhiza Radix was not affected when compared with that of the control group (data not shown), implying that oral administration of the acetonitrile extract of $S$. miltiorrhiza Radix was tolerable to mice at this dosage. However, it was observed that the acetonitrile extract of $S$. miltiorrhiza Radix significantly decreased the incidence (Fig. 5B) and volume (P<0.05; Fig. 5C) of PC-3 xenografts in mice after 4 weeks of administration compared with the untreated control. At autopsy, it was observed that the acetonitrile extract of S. miltiorrhiza Radix decreased the overall incidence (Fig. 5D, left panel) of PC-3 xenografts in nude mice. Furthermore, the weight of PC-3 xenografts in nude mice was significantly decreased using the acetonitrile extract of $S$. miltiorrhiza Radix compared with the untreated control $(\mathrm{P}<0.05$; Fig. 5D, right panel). These results indicate 
that the acetonitrile extract of $S$. miltiorrhiza Radix inhibits the incidence and growth of prostate cancer in vivo.

\section{Discussion}

In the present study, an MTT assay with 400 traditional medicinal plants, extracted with water or acetonitrile, was performed and it was identified that the acetonitrile extract of S. miltiorrhiza Radix exhibited marked growth-inhibitory effects on PC-3 cells. In addition, it was observed that the inhibition of PC-3 cell growth by the acetonitrile extract of S. miltiorrhiza Radix occurred through the induction of cell cycle arrest and apoptosis via the generation of intracellular ROS. A previous study has revealed that $>50$ chemical ingredients are present in S. miltiorrhiza Radix, including hydrophilic phenolic acids and lipophilic tanshionones (12). As $S$. miltiorrhiza Radix extracted using acetonitrile, but not using water, exerted significant growth-inhibitory effects, it may be that lipophilic constituents, rather than hydrophilic compounds, in S. miltiorrhiza Radix contributed to the growth inhibition of PC-3 cells. Consistent with this hypothesis, two previous studies have demonstrated that lipophilic tanshinones exhibited growth-inhibitory effects on prostate cancer cells, including PC-3 cells $(13,14)$. However, the amount of lipophilic tanshinones that exist in the acetonitrile extract of S. miltiorrhiza Radix remains unclear. In addition, it was observed that induction of apoptosis by the acetonitrile extract of S. miltiorrhiza Radix occurred exclusively through the extrinsic apoptotic signaling pathway. Although the underlying molecular mechanism by which the acetonitrile extract of S. miltiorrhiza Radix induces apoptosis in PC-3 cells remains unclear, it may be hypothesized that the acetonitrile extract of S. miltiorrhiza Radix lacks phytochemicals mimicking cellular death ligands, including tumor necrosis factor receptor superfamily members 6 and 10, which are able to initiate a death-receptor-initiated signaling pathway in PC-3 cells (15).

It has previously been demonstrated that $S$. miltiorrhiza Radix, also referred to as Danshen in Chinese, exhibits a number of beneficial pharmacological activities. In particular, traditional pharmacological effects of S. miltiorrhiza Radix have been attributed primarily to promoting circulation (16). Therefore, phase III clinical trials for treatment of diabetic retinopathy and heart disease are currently underway with a compound, the 'Danshen dripping pill' (17). However, the number of studies that demonstrate anti-tumorigenic effects of S. miltiorrhiza Radix is fewer, compared with those illustrating its beneficial cardiovascular effects (18). Following the results of the present study demonstrating that the acetonitrile extract of S. miltiorrhiza Radix suppressed the growth of prostate cancer cells in vitro and in vivo, in-depth biochemical studies are required to identify novel ingredients in the acetonitrile extract of S. miltiorrhiza Radix that may be responsible for its anti-tumorigenic effects in prostate cancer.

The present study provides convincing preclinical evidence for anti-tumorigenic effects of the acetonitrile extract of S. miltiorrhiza Radix against the growth of prostate cancer in vitro and in vivo. It was demonstrated that the growth-inhibitory effects of the acetonitrile extract of S. miltiorrhiza Radix against prostate cancer cells occurred through the induction of cell cycle arrest and apoptosis via the generation of intracellular ROS. Considering that a limited number of chemotherapeutic agents useful for treatment of aggressive prostate cancer currently exist, the present study justifies the on-going investigation of the acetonitrile extract of S. miltiorrhiza Radix as a source of a potential chemotherapeutic agent for the treatment of prostate cancer.

\section{Acknowledgements}

The present study was supported by the Gyoenggi Regional Research Center Program of Gyeonggi Province (grant no. GRRC-DONGGUK2015-B02, development and discovery of new therapeutic target modulators).

\section{References}

1. Siegel R, Ma J, Zou Z and Jemal A: Cancer statistics, 2014. CA Cancer J Clin 64: 9-29, 2014.

2. Heidenreich A, Bastian PJ, Bellmunt J, Bolla M, Joniau S, van der Kwast T, Mason M, Matveev V, Wiegel T, Zattoni F, et al: EAU guidelines on prostate cancer. part 1: Screening, diagnosis, and local treatment with curative intent-update 2013. Eur Urol 65: 124-137, 2014.

3. Heidenreich A, Bastian PJ, Bellmunt J, Bolla M, Joniau S, van der Kwast T, Mason M, Matveev V, Wiegel T, Zattoni F, et al: EAU guidelines on prostate cancer. Part II: Treatment of advanced, relapsing and castration-resistant prostate cancer. Eur Urol 65: 467-479, 2014.

4. Saad F and Hotte SJ: Guidelines for the management of castrate-resistant prostate cancer. Can Urol Assoc J 4: 380-384, 2010.

5. Sporn MB and Suh N: Chemoprevention of cancer. Carcinogenesis 21: 525-530, 2000.

6. William WN Jr, Heymach JV, Kim ES and Lippman SM: Molecular targets for cancer chemoprevention. Nat Rev Drug Discov 8: 213-225, 2009.

7. Violette PD and Saad F: Chemoprevention of prostate cancer: Myths and realities. J Am Board Fam Med 25: 111-119, 2012.

8. Burhans WC and Heintz NH: The cell cycle is a redox cycle: Linking phase-specific target to cell fate. Free Radic Biol Med 47: 1282-1293, 2009.

9. Pietenpol JA and Stewart ZA: Cell cycle checkpoint signaling: Cell cycle arrest versus apoptosis. Toxicology 181-182: 475-481, 2002.

10. Circu ML and Aw TY: Reactive oxygen species, cellular redox systems, and apoptosis. Free Radic Biol Med 48: 749-762, 2010.

11. Pompella A, Visvikis A, Paolicchi A, De Tata V and Casini AF: The changing faces of glutathione, a cellular protagonist. Biochem Pharmacol 66: 1499-1503, 2003.

12. Li MH, Chen JM, Peng Y, Wu Q and Xiao PG: Investigation of Danshen and related medicinal plants in China. J Ethnopharmacol 120: 419-426, 2008.

13. Gong Y, Li Y, Lu Y, Li L, Abdolmaleky H, Blackburn GL and Zhou JR: Bioactive tanshinones in Salvia miltiorrhiza inhibit the growth of prostate cancer cells in vitro and in mice. Int J Cancer 129: 1042-1052, 2011.

14. Zhang Y, Won SH, Jiang C, Lee HJ, Jeong SJ, Lee EO, Zhang J, Ye M, Kim SH and Lü J: Tanshinones from Chinese medicinal herb Danshen (Salvia miltiorrhiza Bunge) suppress prostate cancer growth and androgen receptor signaling. Pharm Res 29: 1595-1608, 2012.

15. Elmore S: Apoptosis: A review of programmed cell death. Toxicol Pathol 35: 495-516, 2007.

16. Wu WY and Wang YP: Pharmacological actions and therapeutic applications of Salvia miltiorrhiza depside salt and its active components. Acta Pharmacol Sin 33: 1119-1130, 2012.

17. National Institutes of Health: https://clinicaltrials.gov/ct2/results ?term=NCT00797953\&Search=Search

18. Su CY, Ming QL, Rahman K, Han T and Qin LP: Salvia miltiorrhiza: Traditional medicinal uses, chemistry, and pharmacology. Chin J Nat Med 13: 163-182, 2015. 